\title{
Estudo analítico dos estágios e informações que compõem a compra online
}

\author{
Analytical study of stages and information \\ that constitute online shopping
}

design da informação, Nos últimos anos, o comércio eletrônico tornou-se um ramo promissor e em comércio eletrônico

\author{
André Schlemmer, Stephania Padovani
}

information design, In the past few years, e-commerce became a promising business in constant expansion in Brazilian market, leading consumers to change their way of shopping. Within this context, this study aims to identify the stages and information that compose online shopping in Brazilian e-commerce websites currently in evidence. Through a process analysis, we verified and compared the stages of buying online, which was followed by a graphic-informational analysis to investigate how information has been presented to Brazilian e-consumers. The results showed that the purchase process is changeable and flexible over time, i.e. the interface is adaptable, interfering in methods and ways of navigation. Information and graphic elements found in the analysis fulfill e-consumers needs and requirements for navigating and concretizing online purchasing.

\section{e-commerce}

\section{Introdução}

Nos últimos anos, o comércio eletrônico tornou-se um ramo promissor e em constante expansão no mercado brasileiro. Os dados preveem um crescimento nominal de $25 \%$ ao ano, faturando R $\$ 28$ bilhões em 
2013 (STECANELla, 2013 ). O hábito de realizar compras no Brasil encontra-se em transformação, com os consumidores migrando do comércio tradicional para o online. Apenas no primeiro semestre de 2012, 5,6 milhões de pessoas realizaram a primeira compra online. Consequentemente, o número de e-consumidores aumentou para 37,6 milhões no Brasil (E-BIT, 2012).

Sobre estes aspectos, Albertin (2010) enfatiza que o sistema de comércio eletrônico deve proporcionar ao e-consumidor vantagens de conexão entre a empresa e o usuário, apoio direto na troca de informações, capacidade de eliminar os limites de tempo e espaço e suporte de interatividade para adaptar-se ao comportamento do e-consumidor. Portanto, cumpre aos desenvolvedores preocupar-se além do instrumental, deve-se ouvir e dar importância ao que o e-consumidor tem a dizer sobre suas experiências em comércio eletrônico.

Em nosso estudo, abordamos o tipo de comércio eletrônico B2C (business-to-consumer), termo destinado aos serviços e informações entre empresas produtoras, vendedoras ou prestadoras de serviços e seus consumidores (TASSABEHJI, 2003; QIN, 2009).

A escolha e a justificativa do estudo no tipo B2C aconteceram devido aos dados que comprovam a inserção de e-consumidores no Brasil. De acordo com a Braspag (companhia que realiza o processamento de pagamentos para comércio eletrônico na América Latina) o Brasil apresentou em 2012, um acréscimo de $46 \%$ de volume de transações no varejo online ao comparar com 2011, comprovando a expansão do comércio eletrônico no país (BRASPAG, 2012). A tendência ao crescimento continua presente em 2013, segundo o estudo realizado pelo IDV (Instituto para Desenvolvimento do Varejo) a estimativa do comércio eletrônico brasileiro será de $35 \%$ de aumento comparado com 2012 (INFO ONLINE, 2012).

Constatamos através destes dados que o consumidor tende a modificar cada vez mais seus hábitos de comprar, ou seja, substituindo a compra tradicional para a online. Sendo assim, surgem os questionamentos do nosso estudo: quais são os estágios para comprar online e como as informações têm sido apresentadas aos e-consumidores?

Este artigo tem por objetivo analisar e comparar, com base na literatura, os estágios para comprar online, como também, verificar como as informações estão sendo apresentadas na interface do comércio eletrônico. Para tanto, analisamos onze sites em destaque no Brasil utilizando fluxograma do processo de compra online e modelo de análise gráfico-informacional. 


\section{0 processo da compra em comércio eletrônico B2C}

Durante a revisão de literatura, encontramos três autores principais, que apresentaram em seus estudos o processo da compra em comércio eletrônico.

Segundo O'Connell (2002), o processo inicia-se no momento que e-consumidor acessa um site $\mathrm{B} 2 \mathrm{C}$, por intermédio de uma página inicial. Na página inicial o e-consumidor pode realizar a escolha de um bem, produto ou serviço, através de um menu; Após a escolha feita pelo e-consumidor, o sistema direciona para o carrinho de compras, então o e-consumidor confere as informações da compra; Após realizar as compras desejadas, o e-consumidor avança para o processo de encaminhamento do pedido, aprovação da compra, entrega e definição da forma de pagamento.

No estudo de Miranda (2004), o processo foi composto por 6 estágios: A página inicial, apresentam-se as informações pertinentes do que o site pode oferecer aos e-consumidores. A localização do produto, neste estágio o e-consumidor busca as informações através das ferramentas de busca ou pelas categorias apresentadas no menu. A lista de produtos, que apresenta a especificação do produto, neste estágio o e-consumidor identifica as qualidades e informações pertinentes do produto que satisfaça a sua necessidade. O quarto estágio chama-se avaliação do produto, destinado a comparar as opções que atendam as necessidades do e-consumidor, neste estágio formam as opiniões, atitudes e intenções das alternativas apresentadas no produto. Na sequência, o checkout (ou processo de finalização de compra), apresenta todas as informações do produto colocado no carrinho de compras e o e-consumidor poderá ver as informações de prazo de entrega e o valor do frete. O sexto estágio definido como recebimento e aceitação do produto, estágio da avaliação pós-compra, ou seja, o produto atendeu as expectativas do e-consumidor? O produto teve o desempenho favorável?

Em seu estudo, Junior (2006) definiu três situações diferentes para o processo de busca e compra de um produto, ou seja, pode ser feita através de um produto específico, por gênero ou livre. Na sequência, apresentamos para exemplificação, uma adaptação sintetizada com os estágios principais de um dos fluxogramas criados por Junior (2006), o fluxograma escolhido foi o da busca livre e compra de um produto. 


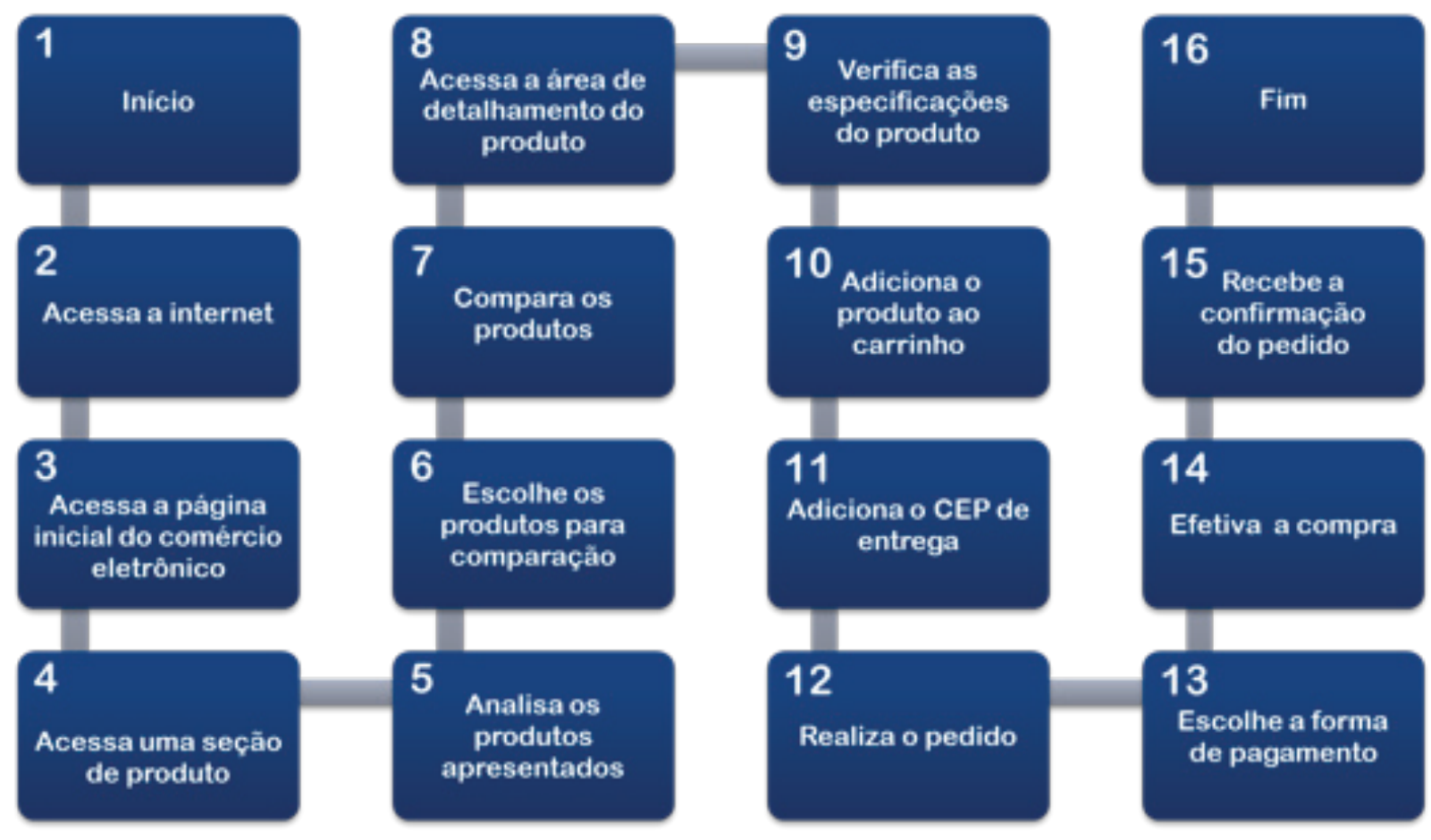

Figura 1 Fluxograma de busca livre e compra de um produto (Fonte: Adaptado de Junior (2006))

No que se refere a uma configuração geral do processo, podemos observar por uma comparação dos estudos, a existência de estágios principais listados por todos os autores, mas também, estão presentes em cada estudo, estágios específicos. O fluxograma de compra livre de Junior (2006) mostra em detalhes cada passo que o e-consumidor encontra no processo de compra, já no estudo de Miranda (2004) o processo foi sintetizado, as etapas finais da realização da compra foram representadas em um único estágio, definido como Checkout, no caso de O'Connel aparecem divididas apenas em entrega e pagamento.

Ao comparar os modelos do processo de compra, podemos observar que O'Connel (2002) citou o encaminhamento do pedido e aprovação da compra, estágios que não aparecem no modelo de Miranda (2004), pois a autora enfatizou no seu processo o produto, valorizando o recebimento e aceitação das expectativas e do desempenho favorável ao e-consumidor.

Durante a comparação, encontramos diferenças nos estágios finais do processo da compra, o fluxograma de Junior (2006) não cita a forma de entrega, apenas o e-consumidor digita o CEP e realiza o pedido, no caso de O'Connel (2002) o e-consumidor encontra o estágio forma de entrega e pagamento, para Miranda (2004) tudo fica sintetizado no Checkout. Portanto, pode-se entender que houve mudanças no processo, pois os estudos não foram realizados no mesmo tempo. 


\section{O sistema de informação em comércio eletrônico B2C}

No âmbito do comércio eletrônico, o sistema informacional é "responsável pela apresentação ou acesso às informações que são necessárias ao esclarecimento dos usuários no exato momento em que são solicitadas, auxiliando-o a tomarem suas decisões de forma consciente, rápida e segura" (JUNIOR, 2006, p. 77). O mesmo autor propôs um modelo conceitual com base nos requisitos informacionais e nas ferramentas que auxiliam o processo de compra online.

O modelo de Junior (2006) é divido em cinco macro-setores: página inicial, seção do produto, área de detalhamento do produto, carrinho de compras e a finalização da compra. Cada macro setor tem uma subdivisão em: informações, acesso às informações, ferramentas e acesso às ferramentas. Por fim, os elementos são classificados em: obrigatório, necessário ou desejável.

Como visto, o comércio eletrônico é constituído por informações e ferramentas para suprir necessidades e esclarecimentos durante a utilização do comércio eletrônico. Porém, como as informações e ferramentas são apresentadas na interface?

Uma interface precisa ser simplesmente, uma extensão da pessoa, ou seja, o sistema e seu software devem refletir as capacidades de uma pessoa e responder suas necessidades específicas (GALITZ, 2002). Para Fleming (1998, p. 64) "a interface é o intermediário entre usuários e conteúdo, um interprete e guia para as complexidades de um site". Também pode ser compreendida como uma coleção de objetos, ferramentas, linguagens que se apresenta entre as pessoas e as máquinas que pretende utilizar, por exemplo, telas de um computador (LANSDALE E ORMEROD, 1994).

A partir dessas conceituações, podemos concluir que para os e-consumidores utilizarem o comércio eletrônico é necessário à interface do site fornecer objetos, ferramentas, linguagem que estabeleçam a interação. Portanto, através de estudos anteriores (e.g.: FLEMING, 1998; GALITZ, 2002; CYBIS, 2003; ROBBINS, 2006; CYBIS, BETIOL E FAUST, 2007; KALBACH, 2007; TIDWELL, 2011; WEBOPEDIA, 2012 e MATHWORKS, 2012), conseguimos definir os componentes da interface que auxiliam a navegação do e-consumidor pelo site. Os componentes foram dividimos em duas categorias: objetos de interação e recursos audiovisuais.

Objetos de interação: os objetos de interação são definidos como representações geradas pelas relações estabelecidas entre janelas, menus, botões, caixas de diálogo, etc., sobre as ações do e-consumidor em uma perspectiva funcional-estrutural (СувіS, 2003; СувіS, Betiol E FAUST, 2007). Podemos dizer que objetos de interação são componentes da interface que auxiliam a navegação do e-consumidor pelo site, para encontrar informações e chegar aos objetivos.

Recursos audiovisuais: Os recursos audiovisuais são definidos como representações simbólicas geradas pelas relações estabelecidas entre imagens, elementos textuais, ícones, som, animações, elementos 
esquemáticos, etc., que transmitem significados ao e-consumidor (CYBIS, 2003; CYBIS, BETIOL E FAUST, 2007). Os recursos audiovisuais criam uma lógica visual que facilita a navegação e fornece o entendimento do conteúdo ao e-consumidor (KALBACH, 2007).

\section{Método}

O estudo analítico foi composto pela técnica de análise de processo, com o intuito de comparar e descrever os estágios para realizar uma compra online e análise gráfico-informacional para verificar quais informações têm sido apresentadas aos e-consumidores brasileiros.

A amostra foi composta por 11 sites B2C que estão em destaque no Brasil atualmente em três fontes especializadas na divulgação de resultados sobre o comércio eletrônico: Top of mind internet 2012 ; E-commercebrasil.org e Alexa The Web Information Company (vide tabela 1).

Tabela 1 Sites B2C analisados no estudo

\begin{tabular}{|c|c|c|}
\hline Sites B2C & Categoria & $\begin{array}{l}\text { Frequência de } \\
\text { atualização }\end{array}$ \\
\hline http://www.netshoes.com.br & Calçados, esportes e derivados & \multirow[t]{11}{*}{ Diariamente } \\
\hline http://www.livrariasaraiva.com.br & Livraria online & \\
\hline http://www.voegol.com.br & Passagens aéreas & \\
\hline http://www.americanas.com.br & \multirow[t]{8}{*}{ Vários departamentos } & \\
\hline http://www.submarino.com.br & & \\
\hline http://www.pontofrio.com.br & & \\
\hline http://www.magazineluiza.com.br & & \\
\hline http://www.extra.com.br & & \\
\hline http://www.comprafacil.com.br & & \\
\hline http://www.casasbahia.com.br & & \\
\hline http://www.walmart.com.br & & \\
\hline
\end{tabular}

Inicialmente, realizamos a análise de processo para saber quais estágios compunham os sites $\mathrm{B} 2 \mathrm{C}$. O método de análise de processo, no âmbito do design de interface, condiz com uma sequência de tarefas que os usuários devem realizar ou são prováveis de realizar em uma determinada ordem (HACKOS E REDISH, 1998).

Sendo assim, para definirmos quais eram os estágios do processo de compra, que seriam então, analisados e comparados, elaboramos um fluxograma baseado nos estudos de O'Connel (2002), Miranda (2004) e Junior (2006): 


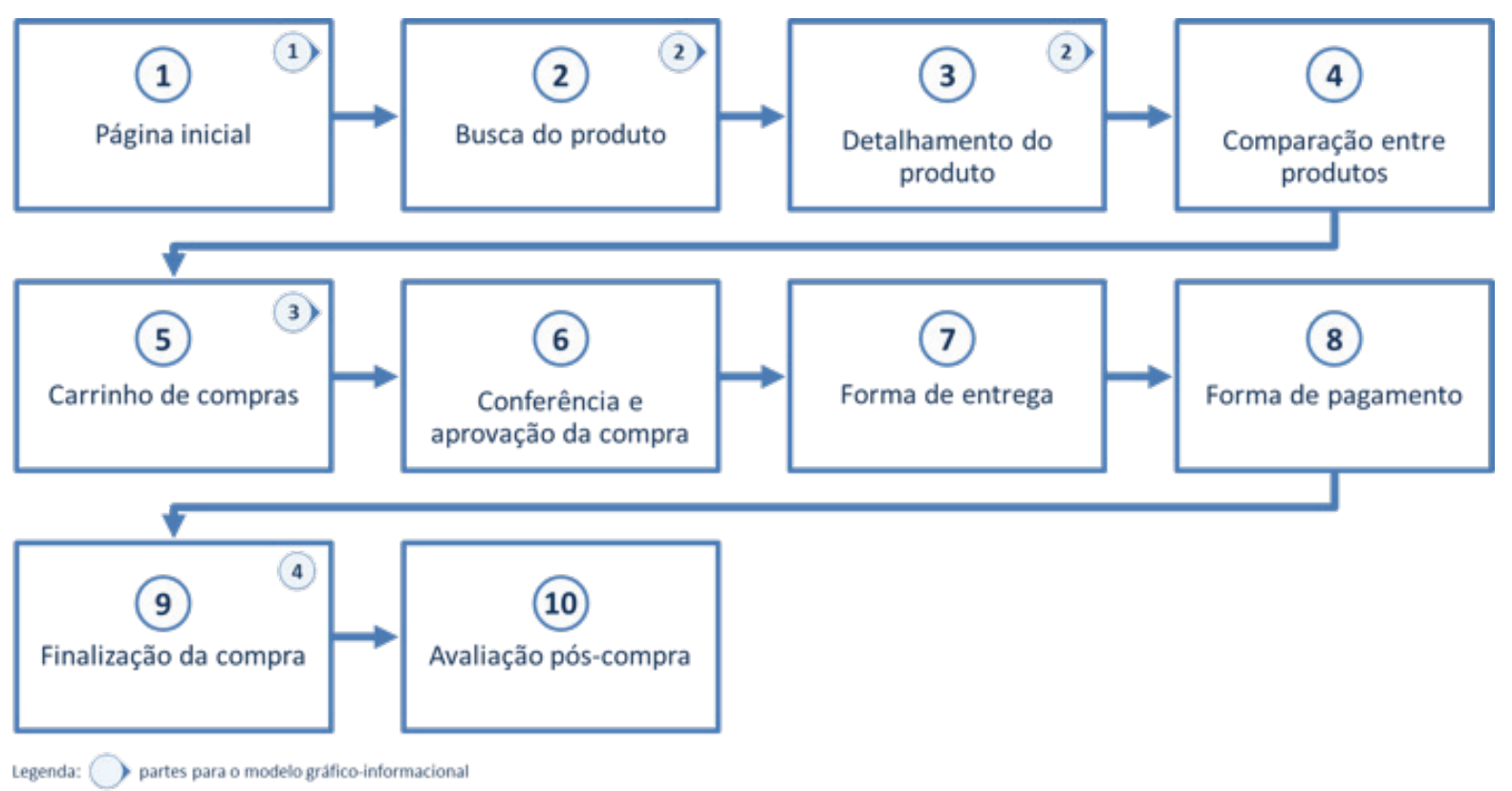

Figura 2 Fluxograma do processo de compra online (Fonte: Baseado em O'Connel (2002); Miranda (2004) e Junior (2006))

Na segunda parte do estudo, análise gráfico-informacional, analisaram-se os mesmos 11 sites $\mathrm{B} 2 \mathrm{C}$, utilizando-se para tal, modelo de análise gráfico-informacional baseado em Cybis (2003), Junior (2006) e Padovani, Spinillo e Gomes (2009). A análise gráficoinformacional foi dividida em 4 partes (retiradas da análise do processo da compra online), cada qual representando um estágio principal do processo da compra online em $\mathrm{B}_{2} \mathrm{C}$ (vide figura 3).

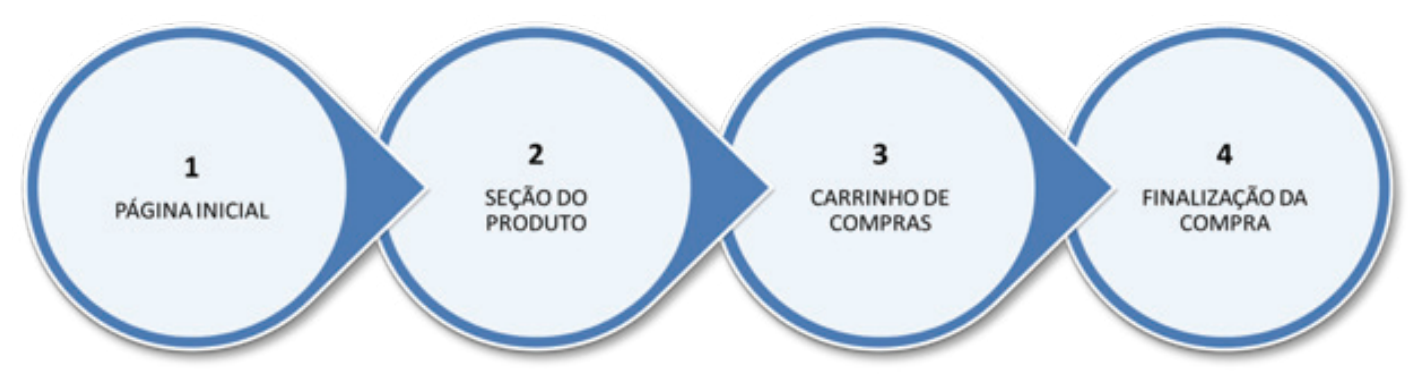

Figura 3 Composição da análise gráfico-informacional

Em cada uma das partes, verificaram-se as informações, os objetos de interação e os recursos audiovisuais (vide figura 4). O modelo de análise gráfico-informacional na íntegra encontra-se em apêndice. 

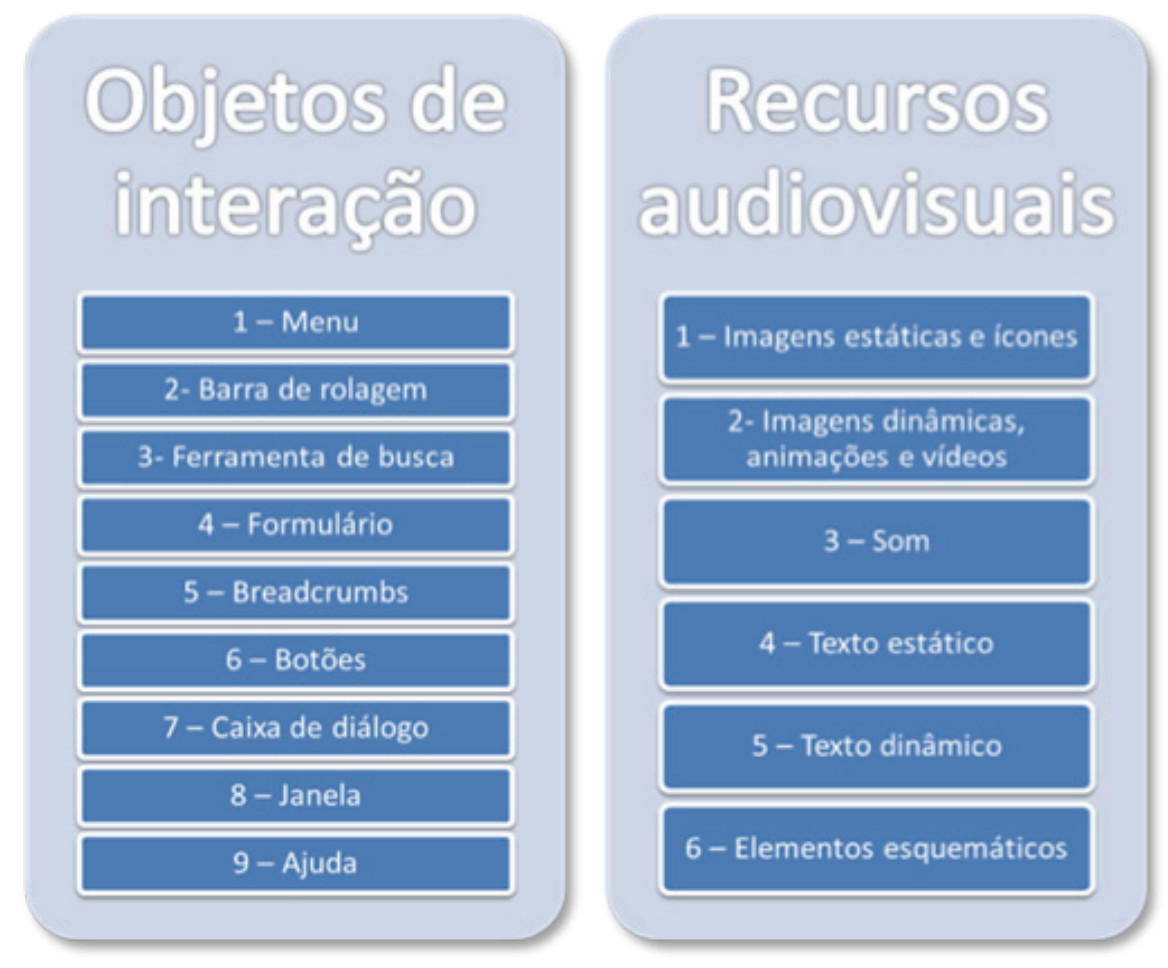

Figura 4 Objetos de interação e recursos audiovisuais da análise gráfica (Fonte: Baseado em Cybis (2003), Junior (2006) e Padovani, Spinillo e Gomes (2009))

\section{Resultados da análise do processo da compra}

De uma maneira geral, a análise do fluxograma dos estágios da compra elaborados a partir dos estudos de O'Connel (2002), Miranda (2004) e Junior (2006) comparados com a nossa amostra, não teve mudanças nos primeiros estágios do processo, ou seja, os estágios permanecem os mesmos. No entanto, constatamos mudanças expressivas no checkout. Acreditamos que estas mudanças ocorreram para diminuir a carga de trabalho, ou seja, minimizar as ações dos e-consumidores durante a finalização da compra. Todavia, deve-se ter cuidado ao modificar o processo, pois o e-consumidor pode encontrar dificuldades durante a navegação e com isso abandonar a compra.

Além de apresentar modificações, houve inclusão de novos estágios, como por exemplo,a identificação (login), como também, exclusão de estágios (ex:. forma de entrega), que não existe mais na maioria dos sites B2C.

Percebemos também que alguns estágios, comparados com os estudos anteriores, sofreram mudanças na sua estrutura. Alguns destes estágios foram mesclados, outros mudaram de posição, foram alterados em sua sequência e alguns já não existem mais no processo atual. Os resultados estão sintetizados a seguir, na tabela 2. 
Tabela 2 Resultados da análise do processo de compra online

\begin{tabular}{|c|c|c|c|c|c|c|c|c|c|c|}
\hline \multirow[b]{4}{*}{ Sites analisados } & \multicolumn{10}{|c|}{ Estágios para efetivar a compra em B2C } \\
\hline & \multicolumn{4}{|c|}{ Etapas iniciais } & \multicolumn{6}{|c|}{ Etapas do Checkout } \\
\hline & 1 & 2 & 3 & 4 & 5 & 6 & 7 & 8 & 9 & 10 \\
\hline & 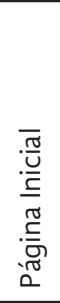 & 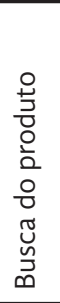 & 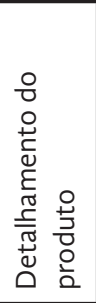 & 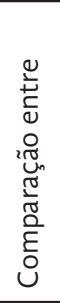 & 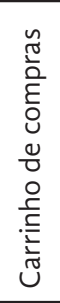 & 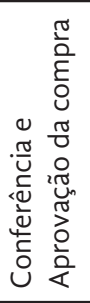 & 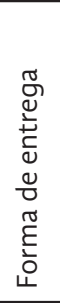 & 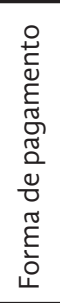 & 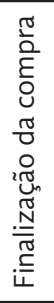 & 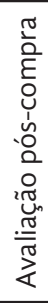 \\
\hline Americanas & & & & $x$ & & $\sqrt{ }$ & x & 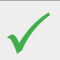 & $\checkmark$ & x \\
\hline Submarino & & & & $\mathbf{x}$ & & $\mathbf{x}$ & $\sqrt{ }$ & & $x$ & $x$ \\
\hline Netshoes & & & & $x$ & & $x$ & $\checkmark$ & & $x$ & $x$ \\
\hline Pontofrio & & & & & & $x$ & $x$ & & $x$ & $x$ \\
\hline Magazineluiza & & & & & & $x$ & $\mathbf{x}$ & & $x$ & $\mathbf{x}$ \\
\hline Extra & & & & $\sqrt{ }$ & & $\mathbf{x}$ & $\mathbf{x}$ & & $\mathbf{x}$ & $\mathbf{x}$ \\
\hline Comprafacil & & & & $x$ & $\sqrt{ }$ & $\mathbf{x}$ & $x$ & & $\mathbf{x}$ & $x$ \\
\hline Casasbahia & & & & $\sqrt{ }$ & $\sqrt{ }$ & $x$ & $x$ & & $x$ & $x$ \\
\hline Walmart & $\sqrt{ }$ & & 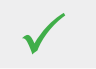 & $x$ & $\checkmark$ & $x$ & $\checkmark$ & & $x$ & $x$ \\
\hline Livrariasaraiva & $V$ & & & $x$ & 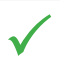 & $\checkmark$ & $x$ & & $\checkmark$ & $x$ \\
\hline Voegol & $\checkmark$ & 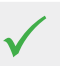 & $\sqrt{ }$ & $x$ & $x$ & $\sqrt{ }$ & $x$ & $v$ & $x$ & $x$ \\
\hline Total de ocorrências & 11 & 11 & 11 & 4 & 10 & 3 & 3 & 11 & 2 & 0 \\
\hline
\end{tabular}

A partir disso, concluímos que o processo da compra é mutável, flexível ao longo do tempo de existência nos sites B2C, ou seja, a interface é adaptável, pois as tecnologias que movem este serviço vivem em constante mudança, ocasionadas por suas evoluções, inovações que acaba interferindo nos métodos e formas de navegação e por isso modificam os hábitos dos e-consumidores.

Em suma, os resultados da nossa análise serviram para comparar como estava sendo feito o processo de efetivação da compra online por meio da literatura e como o processo está sendo feito atualmente. Em uma comparação breve, concluímos que houve mudanças na sequência dos estágios, aparecendo tanto inclusão quanto exclusão, como também, apresentou ordem diferenciada, estágio facultativo e ainda diferenciação por gênero específico, ao comparar com os estudos de O'Connel (2002), Miranda (2004) e Junior (2006).

Diante do exposto, podemos dizer que os estágios para realizar a compra online não são mais os mesmos dos estudos anteriores, tiveram modificações ao longo do tempo, comprovado pela comparação feita nesta amostra. Atualmente, o processo da compra 
ocorre de maneira semelhante entre os sites analisados, existindo um padrão sequencial dos principais estágios, com isso, apresentamos o novo fluxograma dos estágios da compra online.

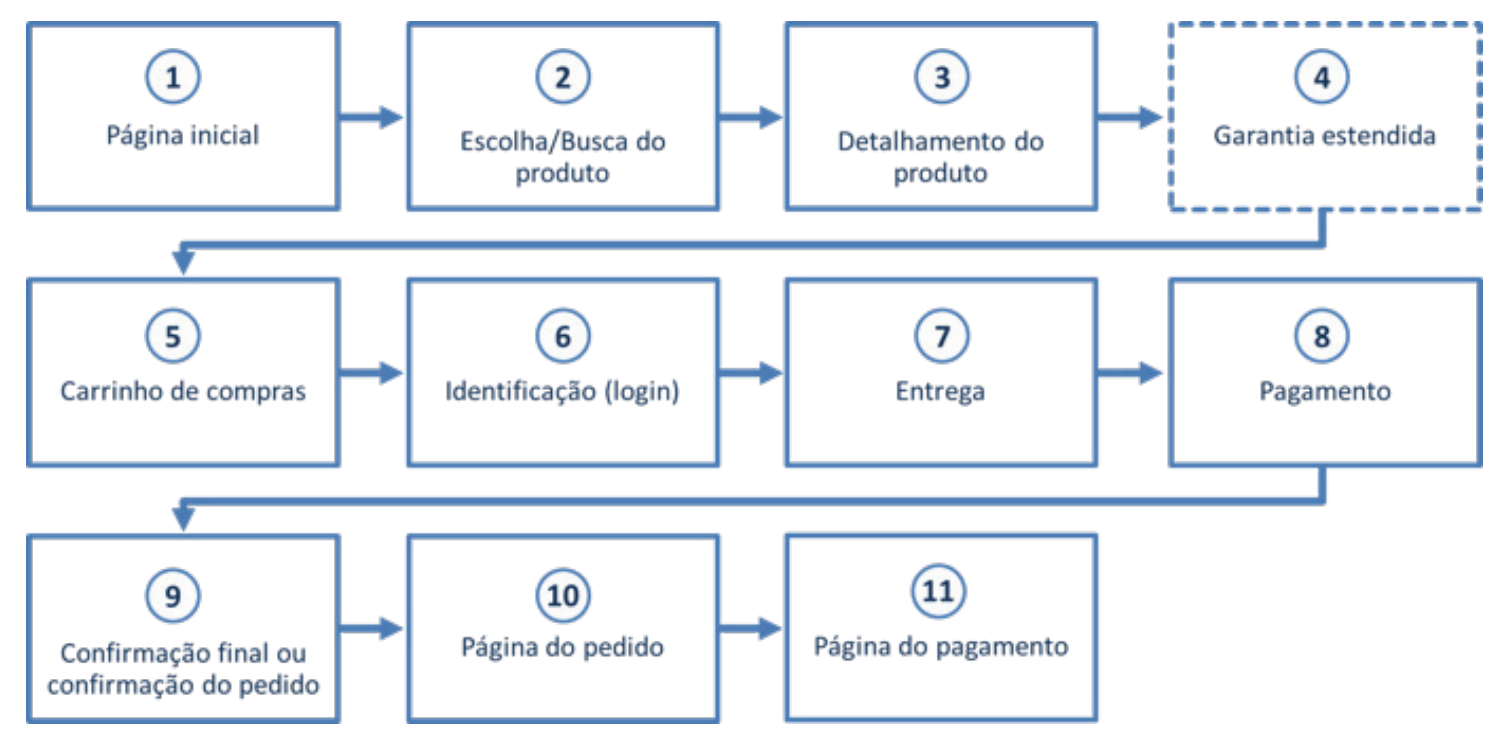

Figura 5 Fluxograma do processo de compra online (atual)

Sobre o novo fluxograma do processo de compra, cabe ressaltar que o estágio da garantia estendida, é uma modalidade que complementa a garantia do produto ou estende, amplia está garantia para o e-consumidor. Sendo assim, este estágio não é destinado a todos os produtos que estão disponíveis nos sites B2C, ou seja, pode não aparecer em alguns casos, onde o valor ou tipo de produto não é compatível com a modalidade. Também, deixamos esclarecido que a nossa análise foi realizada até o estágio da confirmação final ou confirmação do pedido, mesmo assim, identificamos que existe o estágio da página do pedido e o estágio da página do pagamento em nossa amostra.

Podemos constatar que o processo da compra sofre mudanças também ao se referir ao gênero de $\mathrm{B} 2 \mathrm{C}$ que o e-consumidor realizará sua compra. No gênero de passagens aéreas, por exemplo, apresenta-se a parte inicial da mesma forma que outros sites analisados, começando pela página inicial, depois, escolha/busca do produto, no caso específico, escolha do voo e em seguida, seus dados, parte destinada a identificação. A diferenciação aparece no estágio da escolha da poltrona, um estágio novo no processo. O estágio é destinado ao preenchimento dos extras da viagem, o checkout é similar aos outros sites e procede nos estágios pagamento, resumo da compra e confirmação. Todavia, percebemos que quando se modifica o gênero de B2C, também muda-se o contexto dos elementos na interface, sua nomenclatura e estrutura, possivelmente, para facilitar a navegação da compra online. 


\section{Resultados da análise gráfico-informacional}

A seguir apresentamos uma síntese com todos os itens que compunham a análise informacional, separados por estágio.

Tabela 3 Síntese dos resultados da análise informacional

\begin{tabular}{|c|c|c|c|c|c|c|c|c|c|c|c|c|}
\hline \multicolumn{13}{|c|}{ RESULTADOS DA ANÁLISE INFORMACIONAL } \\
\hline \multirow[b]{2}{*}{ Itens da análise } & \multicolumn{12}{|c|}{ Sites analisados } \\
\hline & 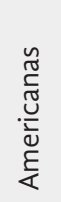 & 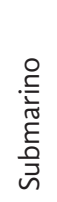 & 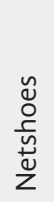 & $\begin{array}{l}\stackrel{0}{+2} \\
\stackrel{2}{0} \\
\stackrel{0}{0} \\
0\end{array}$ & $\begin{array}{l}\frac{\pi}{N} \\
\frac{N}{\tilde{J}} \\
\cdot \frac{\bar{N}}{N} \\
\widetilde{N} \\
\Sigma\end{array}$ & 䒯 & 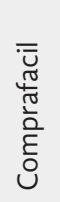 & 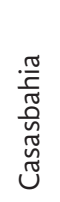 & $\frac{\sqrt[t]{\frac{1}{E}}}{3}$ & 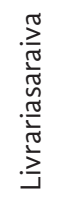 & $\begin{array}{l}\overline{\text { o }} \\
\text { Оे } \\
\text { > }\end{array}$ & 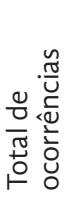 \\
\hline \multicolumn{13}{|l|}{ PÁGINA INICIAL } \\
\hline Informações da empresa & $\checkmark$ & $\mathrm{P}$ & $\mathbf{P}$ & $\checkmark$ & $\checkmark$ & $\checkmark$ & $\mathbf{P}$ & $\checkmark$ & $\mathbf{x}$ & $\checkmark$ & $\mathbf{P}$ & 6 \\
\hline Informações de contato & $\checkmark$ & $\checkmark$ & $\checkmark$ & $\checkmark$ & $\checkmark$ & $\checkmark$ & $\checkmark$ & $\checkmark$ & $\checkmark$ & $\checkmark$ & $\checkmark$ & 11 \\
\hline Políticas de segurança e privacidade & $\checkmark$ & $\checkmark$ & $\checkmark$ & $\checkmark$ & $\checkmark$ & $\checkmark$ & $\checkmark$ & $\checkmark$ & $\checkmark$ & $\checkmark$ & $\checkmark$ & 11 \\
\hline Políticas de troca e devolução & $\checkmark$ & $\checkmark$ & $\checkmark$ & $\checkmark$ & $\checkmark$ & $\checkmark$ & $\checkmark$ & $\checkmark$ & $\checkmark$ & $\checkmark$ & $\mathbf{x}$ & 10 \\
\hline Listagem dos departamentos & $\checkmark$ & $\checkmark$ & $\checkmark$ & $\checkmark$ & $\checkmark$ & $\checkmark$ & $\checkmark$ & $\checkmark$ & $\checkmark$ & $\checkmark$ & $\checkmark$ & 11 \\
\hline Visualização do meu carrinho & $\checkmark$ & $\checkmark$ & $\checkmark$ & $\checkmark$ & $\checkmark$ & $\checkmark$ & $\checkmark$ & $\checkmark$ & $\checkmark$ & $\checkmark$ & $x$ & 10 \\
\hline Visualização de promoções & $\checkmark$ & $\checkmark$ & $\checkmark$ & $\checkmark$ & $\checkmark$ & $\checkmark$ & $\checkmark$ & $\checkmark$ & $\checkmark$ & $\checkmark$ & $\checkmark$ & 11 \\
\hline Visualização de lançamentos/destaques & $\checkmark$ & $\checkmark$ & $\checkmark$ & $\checkmark$ & $\checkmark$ & $\checkmark$ & $\checkmark$ & $\checkmark$ & $\checkmark$ & $\checkmark$ & $\checkmark$ & 11 \\
\hline \multicolumn{13}{|l|}{ SEÇÃO DO PRODUTO } \\
\hline Visualização do produto com detalhes e preço & $\checkmark$ & $\checkmark$ & $\checkmark$ & $\checkmark$ & $\checkmark$ & $\checkmark$ & $\checkmark$ & $\checkmark$ & $\checkmark$ & $\checkmark$ & $\checkmark$ & 11 \\
\hline Informações técnicas avançadas & $\checkmark$ & $\checkmark$ & $\checkmark$ & $\checkmark$ & $\checkmark$ & $\checkmark$ & $\checkmark$ & $\checkmark$ & $\checkmark$ & $\checkmark$ & $\checkmark$ & 11 \\
\hline Custo de frete durante a seleção do produto & $\checkmark$ & $\checkmark$ & $x$ & $\checkmark$ & $x$ & $\checkmark$ & $\checkmark$ & $\checkmark$ & $\checkmark$ & $\mathbf{x}$ & x & 7 \\
\hline Prazo de entrega durante a seleção do produto & $\checkmark$ & $\checkmark$ & $x$ & $\checkmark$ & $x$ & $\checkmark$ & $\checkmark$ & $\checkmark$ & $\checkmark$ & $\checkmark$ & $x$ & 8 \\
\hline \multicolumn{13}{|l|}{ CARRINHO DE COMPRAS } \\
\hline Visualização dos dados do produto & $\checkmark$ & $\checkmark$ & $\checkmark$ & $\checkmark$ & $\checkmark$ & $\checkmark$ & $\checkmark$ & $\checkmark$ & $\checkmark$ & $\checkmark$ & $x$ & 10 \\
\hline $\begin{array}{l}\text { Visualização do preço unitário e valor total } \\
\text { de cada produto }\end{array}$ & $\checkmark$ & $\checkmark$ & $\checkmark$ & $\checkmark$ & $\checkmark$ & $\checkmark$ & $\checkmark$ & $\checkmark$ & $\checkmark$ & $\checkmark$ & $\mathbf{x}$ & 10 \\
\hline Listagem dos produtos adicionados & $\checkmark$ & $\checkmark$ & $\checkmark$ & $\checkmark$ & $\checkmark$ & $\checkmark$ & $\checkmark$ & $\checkmark$ & $\checkmark$ & $\checkmark$ & $x$ & 10 \\
\hline Identificação de descontos fornecidos & $\checkmark$ & $\checkmark$ & $\checkmark$ & $\checkmark$ & $\checkmark$ & $\checkmark$ & $\checkmark$ & $\checkmark$ & $\checkmark$ & $\checkmark$ & $\mathrm{x}$ & 10 \\
\hline Custo final da compra & $\checkmark$ & $\checkmark$ & $\checkmark$ & $\checkmark$ & $\checkmark$ & $\checkmark$ & $\checkmark$ & $\checkmark$ & $\checkmark$ & $\checkmark$ & $x$ & 10 \\
\hline \multicolumn{13}{|l|}{ FINALIZAÇÃO DA COMPRA } \\
\hline Visualização da linha do tempo & $\checkmark$ & $\checkmark$ & $\checkmark$ & $x$ & $\checkmark$ & $x$ & $\checkmark$ & $x$ & $\checkmark$ & $\checkmark$ & $\checkmark$ & 8 \\
\hline Forma de pagamento & $\checkmark$ & $\checkmark$ & $\checkmark$ & $\checkmark$ & $\checkmark$ & $\checkmark$ & $\checkmark$ & $\checkmark$ & $\checkmark$ & $\checkmark$ & $\checkmark$ & 11 \\
\hline Tipos de entrega & $x$ & $x$ & $\checkmark$ & $x$ & $x$ & $x$ & $x$ & $x$ & $\checkmark$ & $\checkmark$ & $x$ & 3 \\
\hline Prazo de entrega & $\checkmark$ & $\checkmark$ & $\checkmark$ & $\checkmark$ & $\checkmark$ & $\checkmark$ & $\checkmark$ & $\checkmark$ & $\checkmark$ & $\checkmark$ & x & 10 \\
\hline Garantia do produto & LI & LI & LI & LI & LI & LI & LI & LI & LI & LI & $x$ & 0 \\
\hline $\begin{array}{l}\text { Resumo dos produtos comprados em } \\
\text { conjunto com o custo final da compra }\end{array}$ & $\checkmark$ & $\checkmark$ & $\checkmark$ & $\checkmark$ & $P$ & $\checkmark$ & $\checkmark$ & $\checkmark$ & $\checkmark$ & $\checkmark$ & $\checkmark$ & 10 \\
\hline $\begin{array}{l}\text { Todas as informações (dados pessoais, } \\
\text { entrega, valor e forma de pgto) para } \\
\text { confirmação da compra }\end{array}$ & $\checkmark$ & $\mathbf{x}$ & $x$ & $x$ & $x$ & $x$ & $x$ & $x$ & $x$ & $\checkmark$ & $\checkmark$ & 3 \\
\hline
\end{tabular}

Legenda: $\checkmark=$ existe $\mid \boldsymbol{X}=$ não existe $\mid \mathrm{P}=$ parcial $\mid \mathbf{L I}=$ localização inadequada

Infodesıgn | São Paulo | v. 10 | n. 3 [2013], p. 224-244 
A partir dos resultados da análise informacional, podemos constatar que a maioria dos sites apresentou todas as informações recomendadas pela literatura nos estágios da página inicial, seção do produto e carrinho de compras. Em contraste, no estágio da finalização da compra encontramos poucas informações recomendadas pela literatura. Destaque para o item tipos de entrega, onde apenas 3 dos 11 sites da amostra forneciam opções ao e-consumidor, exemplificado a seguir, na figura 6.

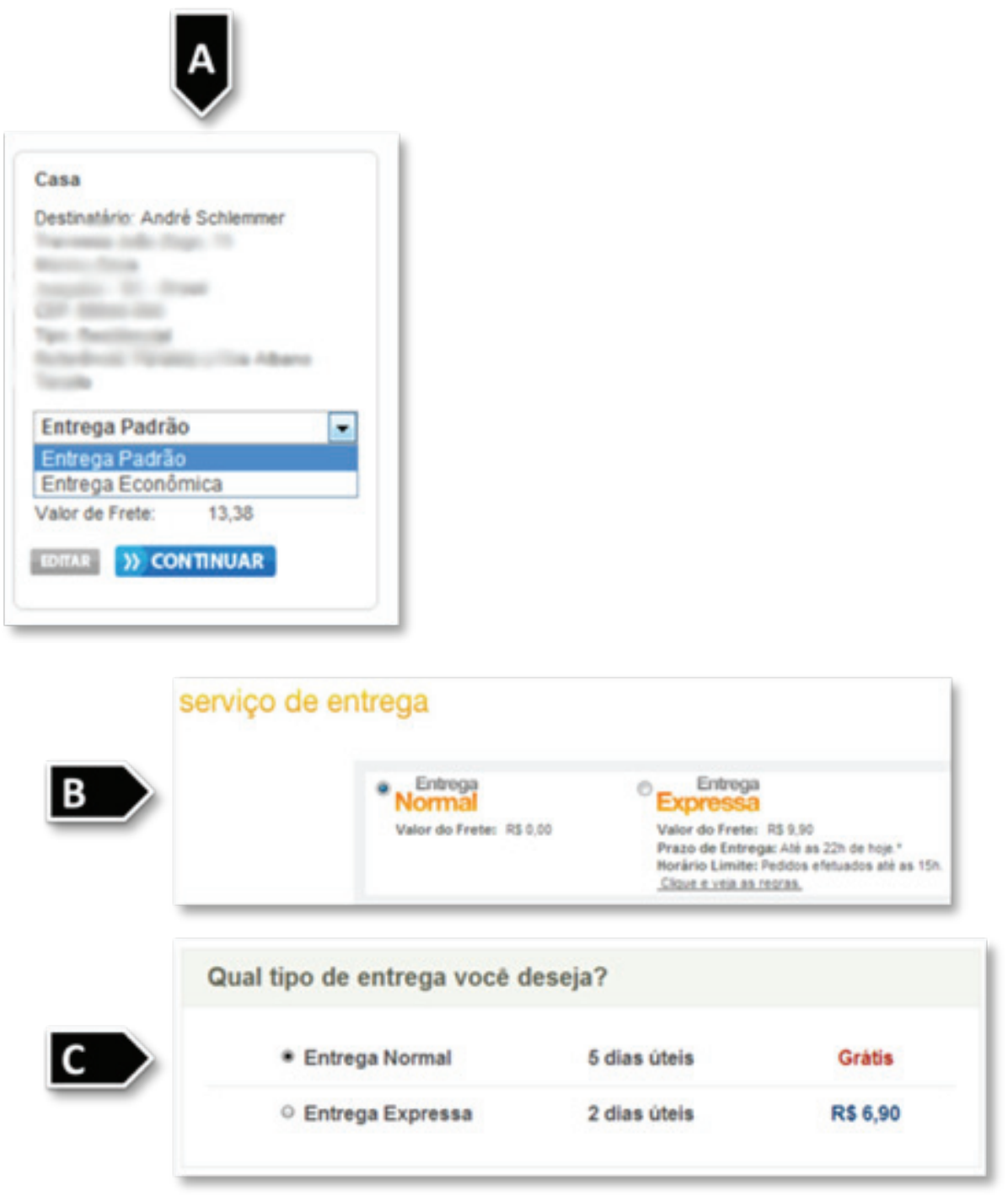

Figura 6 Tipos de entrega

Exemplo A: Entrega padrão ou econômica - http://www.walmart.com. br (16/0412)

Exemplo B: Entrega normal ou expressa - http://www.livrariasaraiva. com.br (19/11/12)

Exemplo C: Entrega normal com frete grátis - http://www.netshoes. com.br (02/11/12) 
A tabela 4 representa a síntese com todos os objetos de interação e recursos audiovisuais encontrados na análise gráfica dos estágios.

Tabela 4 Síntese dos resultados da análise gráfica

\begin{tabular}{|c|c|c|c|c|c|c|c|c|c|c|c|c|c|}
\hline \multicolumn{2}{|c|}{ RESULTADOS DA ANÁLISE GRÁFICA } & AM & SB & NS & PF & ML & EX & CF & $\mathrm{CB}$ & w & LS & VG & TO \\
\hline \multicolumn{14}{|c|}{ PÁGINA INICIAL } \\
\hline \multirow{9}{*}{ 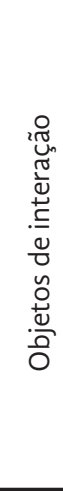 } & 1 - Menu & $\checkmark$ & $\checkmark$ & $\checkmark$ & $\checkmark$ & $\checkmark$ & $\checkmark$ & $\checkmark$ & $\checkmark$ & $\checkmark$ & $\checkmark$ & $\checkmark$ & 11 \\
\hline & 2 - Barra de rolagem & $\checkmark$ & $\checkmark$ & $\checkmark$ & $\checkmark$ & $\checkmark$ & $\checkmark$ & $\checkmark$ & $\checkmark$ & $\checkmark$ & $\checkmark$ & $\checkmark$ & 11 \\
\hline & 3 - Ferramenta de busca & $\checkmark$ & $\checkmark$ & $\checkmark$ & $\checkmark$ & $\checkmark$ & $\checkmark$ & $\checkmark$ & $\checkmark$ & $\checkmark$ & $\checkmark$ & $\checkmark$ & 11 \\
\hline & 4 - Formulário & $\checkmark$ & $\checkmark$ & $\checkmark$ & $\checkmark$ & $\checkmark$ & $\checkmark$ & $\checkmark$ & $\checkmark$ & $\checkmark$ & $x$ & $\checkmark$ & 10 \\
\hline & 5 -Breadcrumbs & $x$ & $x$ & $x$ & $x$ & $x$ & $x$ & $x$ & $x$ & $x$ & $x$ & $x$ & 0 \\
\hline & 6 - Botões & $\checkmark$ & $\checkmark$ & $\checkmark$ & $\checkmark$ & $\checkmark$ & $\checkmark$ & $\checkmark$ & $\checkmark$ & $\checkmark$ & $\checkmark$ & $\checkmark$ & 11 \\
\hline & 7 - Caixa de diálogo & $\checkmark$ & $\checkmark$ & $\checkmark$ & $\checkmark$ & $\checkmark$ & $\checkmark$ & $\checkmark$ & $\checkmark$ & $\checkmark$ & $\checkmark$ & $\checkmark$ & 11 \\
\hline & 8 - Janela & $\checkmark$ & $\checkmark$ & $\checkmark$ & $\checkmark$ & $\checkmark$ & $\checkmark$ & $\checkmark$ & $\checkmark$ & $\checkmark$ & $\checkmark$ & $\checkmark$ & 11 \\
\hline & 9 - Ajuda & $\checkmark$ & $\checkmark$ & $\checkmark$ & $\checkmark$ & $\checkmark$ & $\checkmark$ & $\checkmark$ & $\checkmark$ & $\checkmark$ & $\checkmark$ & $\checkmark$ & 11 \\
\hline \multirow{6}{*}{ 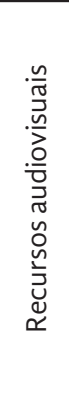 } & $\begin{array}{l}1 \text { - Imagens estáticas e/ou } \\
\text { ícones }\end{array}$ & $\checkmark$ & $\checkmark$ & $\checkmark$ & $\checkmark$ & $\checkmark$ & $\checkmark$ & $\checkmark$ & $\checkmark$ & $\checkmark$ & $\checkmark$ & $\checkmark$ & 11 \\
\hline & $\begin{array}{l}2 \text { - Imagens dinâmicas, } \\
\text { animações e/ou vídeo }\end{array}$ & $\checkmark$ & $\checkmark$ & $\checkmark$ & $\checkmark$ & $\checkmark$ & $\checkmark$ & $\checkmark$ & $\checkmark$ & $\checkmark$ & $\checkmark$ & $\checkmark$ & 11 \\
\hline & 3-Som & $x$ & $x$ & $x$ & $x$ & $x$ & $x$ & $x$ & $x$ & $x$ & $x$ & $x$ & 0 \\
\hline & 4 - Texto estático & $\checkmark$ & $\checkmark$ & $\checkmark$ & $\checkmark$ & $\checkmark$ & $\checkmark$ & $\checkmark$ & $\checkmark$ & $\checkmark$ & $\checkmark$ & $\checkmark$ & 11 \\
\hline & 5 - Texto dinâmico & $\checkmark$ & $\checkmark$ & $\checkmark$ & $x$ & $x$ & $\checkmark$ & $x$ & $x$ & $\checkmark$ & $x$ & $x$ & 5 \\
\hline & 6 - Elementos esquemáticos & $\checkmark$ & $\checkmark$ & $\checkmark$ & $\checkmark$ & $\checkmark$ & $\checkmark$ & $\checkmark$ & $\checkmark$ & $\checkmark$ & $\checkmark$ & $\checkmark$ & 11 \\
\hline \multicolumn{14}{|c|}{ SEÇÃO DO PRODUTO } \\
\hline \multirow{9}{*}{ 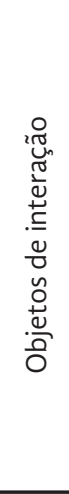 } & 1 - Menu & $\checkmark$ & $\checkmark$ & $\checkmark$ & $\checkmark$ & $\checkmark$ & $\checkmark$ & $\checkmark$ & $\checkmark$ & $\checkmark$ & $\checkmark$ & $\checkmark$ & 11 \\
\hline & 2 - Barra de rolagem & $\checkmark$ & $\checkmark$ & $\checkmark$ & $\checkmark$ & $\checkmark$ & $\checkmark$ & $\checkmark$ & $\checkmark$ & $\checkmark$ & $\checkmark$ & $\checkmark$ & 11 \\
\hline & 3 - Ferramenta de busca & $\checkmark$ & $\checkmark$ & $\checkmark$ & $\checkmark$ & $\checkmark$ & $\checkmark$ & $\checkmark$ & $\checkmark$ & $\checkmark$ & $\checkmark$ & $\checkmark$ & 11 \\
\hline & 4 - Formulário & $x$ & $x$ & $\checkmark$ & $\checkmark$ & $\checkmark$ & $x$ & $\mathbf{x}$ & $\mathrm{x}$ & $x$ & $\checkmark$ & $\mathbf{x}$ & 4 \\
\hline & 5 -Breadcrumbs & $\checkmark$ & $\checkmark$ & $\checkmark$ & $\checkmark$ & $\checkmark$ & $\checkmark$ & $\checkmark$ & $\checkmark$ & $\checkmark$ & $\checkmark$ & $x$ & 10 \\
\hline & 6 - Botões & $\checkmark$ & $\checkmark$ & $\checkmark$ & $\checkmark$ & $\checkmark$ & $\checkmark$ & $\checkmark$ & $\checkmark$ & $\checkmark$ & $\checkmark$ & $\checkmark$ & 11 \\
\hline & 7 - Caixa de diálogo & $\checkmark$ & $\checkmark$ & $\checkmark$ & $\checkmark$ & $\checkmark$ & $\checkmark$ & $\checkmark$ & $\checkmark$ & $\checkmark$ & $\checkmark$ & $x$ & 10 \\
\hline & 8 - Janela & $\checkmark$ & $\checkmark$ & $\checkmark$ & $\checkmark$ & $\checkmark$ & $\checkmark$ & $\checkmark$ & $\checkmark$ & $\checkmark$ & $\checkmark$ & $\checkmark$ & 11 \\
\hline & 9 - Ajuda & $\checkmark$ & $\checkmark$ & $\checkmark$ & $\checkmark$ & $\checkmark$ & $\checkmark$ & $\checkmark$ & $\checkmark$ & $\checkmark$ & $\checkmark$ & $\checkmark$ & 11 \\
\hline \multirow{6}{*}{ 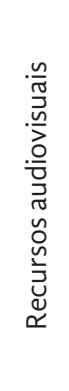 } & $\begin{array}{l}1 \text { - Imagens estáticas e/ou } \\
\text { ícones }\end{array}$ & $\checkmark$ & $\checkmark$ & $\checkmark$ & $\checkmark$ & $\checkmark$ & $\checkmark$ & $\checkmark$ & $\checkmark$ & $\checkmark$ & $\checkmark$ & $\checkmark$ & 11 \\
\hline & $\begin{array}{l}2 \text { - Imagens dinâmicas, } \\
\text { animações e/ou vídeo }\end{array}$ & $\checkmark$ & $\checkmark$ & $\checkmark$ & $\checkmark$ & $\checkmark$ & $\checkmark$ & $\checkmark$ & $\checkmark$ & $\checkmark$ & $\checkmark$ & $x$ & 10 \\
\hline & 3-Som & $\checkmark$ & $\checkmark$ & $\checkmark$ & $x$ & $x$ & $x$ & $\mathrm{x}$ & $\mathrm{x}$ & $\mathbf{x}$ & $\checkmark$ & $x$ & 4 \\
\hline & 4 - Texto estático & $\checkmark$ & $\checkmark$ & $\checkmark$ & $\checkmark$ & $\checkmark$ & $\checkmark$ & $\checkmark$ & $\checkmark$ & $\checkmark$ & $\checkmark$ & $\checkmark$ & 11 \\
\hline & 5 - Texto dinâmico & $x$ & $\checkmark$ & $\checkmark$ & $x$ & $x$ & $x$ & $x$ & $x$ & $x$ & $x$ & $x$ & 2 \\
\hline & 6 -Elementos esquemáticos & $\checkmark$ & $\checkmark$ & $\checkmark$ & $\checkmark$ & $\checkmark$ & $\checkmark$ & $\checkmark$ & $\checkmark$ & $\checkmark$ & $\checkmark$ & $\checkmark$ & 11 \\
\hline
\end{tabular}




\begin{tabular}{|c|c|c|c|c|c|c|c|c|c|c|c|c|c|}
\hline \multicolumn{14}{|c|}{ CARRINHO DE COMPRAS } \\
\hline \multirow{9}{*}{ 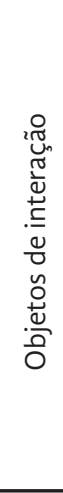 } & $1-$ Menu & $\checkmark$ & $x$ & $\checkmark$ & $\checkmark$ & $\checkmark$ & $\checkmark$ & $\checkmark$ & $\checkmark$ & $x$ & $x$ & $x$ & 7 \\
\hline & 2 - Barra de rolagem & $\checkmark$ & $\checkmark$ & $\checkmark$ & $\checkmark$ & $\checkmark$ & $\checkmark$ & $\checkmark$ & $\checkmark$ & $\checkmark$ & $\checkmark$ & $x$ & 10 \\
\hline & 3 - Ferramenta de busca & $\checkmark$ & $\checkmark$ & $\checkmark$ & $\checkmark$ & $\checkmark$ & $\checkmark$ & $\checkmark$ & $\checkmark$ & $\checkmark$ & $\checkmark$ & $x$ & 10 \\
\hline & 4 - Formulário & $\checkmark$ & $\checkmark$ & $\checkmark$ & $x$ & $x$ & $x$ & $\checkmark$ & $x$ & $x$ & $\checkmark$ & $x$ & 5 \\
\hline & 5 -Breadcrumbs & $x$ & $x$ & $x$ & $x$ & $x$ & $x$ & $x$ & $x$ & $x$ & $x$ & $x$ & $\mathrm{o}$ \\
\hline & 6 - Botões & $\checkmark$ & $\checkmark$ & $\checkmark$ & $\checkmark$ & $\checkmark$ & $\checkmark$ & $\checkmark$ & $\checkmark$ & $\checkmark$ & $\checkmark$ & $x$ & 10 \\
\hline & 7 - Caixa de diálogo & $\checkmark$ & $\checkmark$ & $\checkmark$ & $\checkmark$ & $\checkmark$ & $\checkmark$ & $\checkmark$ & $\checkmark$ & $\checkmark$ & $\checkmark$ & $x$ & 10 \\
\hline & 8 - Janela & $\checkmark$ & $\checkmark$ & $\checkmark$ & $\checkmark$ & $\checkmark$ & $\checkmark$ & $\checkmark$ & $\checkmark$ & $\checkmark$ & $\checkmark$ & $x$ & 10 \\
\hline & 9 - Ajuda & $\checkmark$ & $\checkmark$ & $\checkmark$ & $\checkmark$ & $\checkmark$ & $\checkmark$ & $\checkmark$ & $\checkmark$ & $\checkmark$ & $\checkmark$ & $x$ & 10 \\
\hline \multirow{6}{*}{ 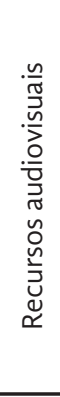 } & $\begin{array}{l}1 \text { - Imagens estáticas e/ou } \\
\text { ícones }\end{array}$ & $\checkmark$ & $\checkmark$ & $\checkmark$ & $\checkmark$ & $\checkmark$ & $\checkmark$ & $\checkmark$ & $\checkmark$ & $\checkmark$ & $\checkmark$ & $x$ & 10 \\
\hline & $\begin{array}{l}2 \text { - Imagens dinâmicas, } \\
\text { animações e/ou vídeo }\end{array}$ & $x$ & $x$ & $\checkmark$ & $x$ & $x$ & $x$ & $x$ & $x$ & $x$ & $x$ & $x$ & 1 \\
\hline & 3-Som & $x$ & $x$ & $x$ & $x$ & $x$ & $x$ & $x$ & $x$ & $x$ & $x$ & $x$ & 0 \\
\hline & 4 - Texto estático & $\checkmark$ & $\checkmark$ & $\checkmark$ & $\checkmark$ & $\checkmark$ & $\checkmark$ & $\checkmark$ & $\checkmark$ & $\checkmark$ & $\checkmark$ & $x$ & 10 \\
\hline & 5 - Texto dinâmico & $x$ & $x$ & $\checkmark$ & $x$ & $x$ & $x$ & $x$ & $x$ & $x$ & $x$ & $x$ & 1 \\
\hline & 6 - Elementos esquemáticos & $\checkmark$ & $\checkmark$ & $\checkmark$ & $\checkmark$ & $\checkmark$ & $\checkmark$ & $\checkmark$ & $\checkmark$ & $\checkmark$ & $\checkmark$ & $x$ & 10 \\
\hline \multicolumn{14}{|c|}{ FINALIZAÇÃO DA COMPRA } \\
\hline \multirow{9}{*}{ 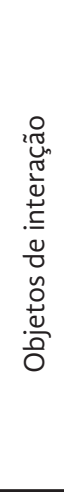 } & 1 - Menu & $x$ & $x$ & $x$ & $x$ & $x$ & $x$ & $x$ & $x$ & $x$ & $x$ & $\checkmark$ & 1 \\
\hline & 2 - Barra de rolagem & $\checkmark$ & $\checkmark$ & $\checkmark$ & $\checkmark$ & $\checkmark$ & $\checkmark$ & $\checkmark$ & $\checkmark$ & $\checkmark$ & $\checkmark$ & $\checkmark$ & 11 \\
\hline & 3 - Ferramenta de busca & $x$ & $x$ & $x$ & $x$ & $x$ & $x$ & $x$ & $x$ & $x$ & $\checkmark$ & $x$ & 1 \\
\hline & 4 - Formulário & $\checkmark$ & $\checkmark$ & $\checkmark$ & $\checkmark$ & $\checkmark$ & $\checkmark$ & $\checkmark$ & $\checkmark$ & $\checkmark$ & $\checkmark$ & $\checkmark$ & 11 \\
\hline & 5 -Breadcrumbs & $x$ & $x$ & $x$ & $x$ & $x$ & $x$ & $x$ & $x$ & $x$ & $x$ & $x$ & $\circ$ \\
\hline & 6 - Botões & $\checkmark$ & $\checkmark$ & $\checkmark$ & $\checkmark$ & $\checkmark$ & $\checkmark$ & $\checkmark$ & $\checkmark$ & $\checkmark$ & $\checkmark$ & $\checkmark$ & 11 \\
\hline & 7 - Caixa de diálogo & $\checkmark$ & $\checkmark$ & $\checkmark$ & $\checkmark$ & $\checkmark$ & $\checkmark$ & $\checkmark$ & $\checkmark$ & $\checkmark$ & $\checkmark$ & $\checkmark$ & 11 \\
\hline & 8 - Janela & $\checkmark$ & $\checkmark$ & $\checkmark$ & $\checkmark$ & $\checkmark$ & $\checkmark$ & $\checkmark$ & $\checkmark$ & $\checkmark$ & $\checkmark$ & $\checkmark$ & 11 \\
\hline & 9 - Ajuda & $\checkmark$ & $\checkmark$ & $\checkmark$ & $\checkmark$ & $\checkmark$ & $\checkmark$ & $\checkmark$ & $\checkmark$ & $\checkmark$ & $\checkmark$ & $\checkmark$ & 11 \\
\hline \multirow{6}{*}{ 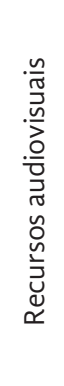 } & $\begin{array}{l}1 \text { - Imagens estáticas e/ou } \\
\text { ícones }\end{array}$ & $\checkmark$ & $\checkmark$ & $\checkmark$ & $\checkmark$ & $\checkmark$ & $\checkmark$ & $\checkmark$ & $\checkmark$ & $\checkmark$ & $\checkmark$ & $\checkmark$ & 11 \\
\hline & $\begin{array}{l}2 \text { - Imagens dinâmicas, } \\
\text { animações e/ou vídeo }\end{array}$ & $x$ & $x$ & $x$ & $x$ & $x$ & $x$ & $\checkmark$ & $x$ & $x$ & $x$ & $x$ & 1 \\
\hline & 3-Som & $x$ & $x$ & $x$ & $x$ & $x$ & $x$ & $x$ & $x$ & $x$ & $x$ & $x$ & o \\
\hline & 4 - Texto estático & $\checkmark$ & $\checkmark$ & $\checkmark$ & $\checkmark$ & $\checkmark$ & $\checkmark$ & $\checkmark$ & $\checkmark$ & $\checkmark$ & $\checkmark$ & $\checkmark$ & 11 \\
\hline & 5 - Texto dinâmico & $x$ & $x$ & $x$ & $x$ & $x$ & $x$ & $x$ & $x$ & $x$ & $x$ & $x$ & 0 \\
\hline & 6 - Elementos esquemáticos & $\checkmark$ & $\checkmark$ & $\checkmark$ & $\checkmark$ & $\checkmark$ & $\checkmark$ & $\checkmark$ & $\checkmark$ & $\checkmark$ & $\checkmark$ & $\checkmark$ & 11 \\
\hline
\end{tabular}

Legenda: $\checkmark=$ existe $\mid \boldsymbol{X}=$ não existe.

Da análise gráfica realizada nos sites da amostra, observamos que os objetos de interação, menu, barra de rolagem, ferramenta de busca, formulário, botões, caixa de diálogo, janela e ajuda estavam presentes em todos os sites da amostra, e a opção de breadcrumbs foi encontrada na maioria dos sites da amostra (10 de 11). 


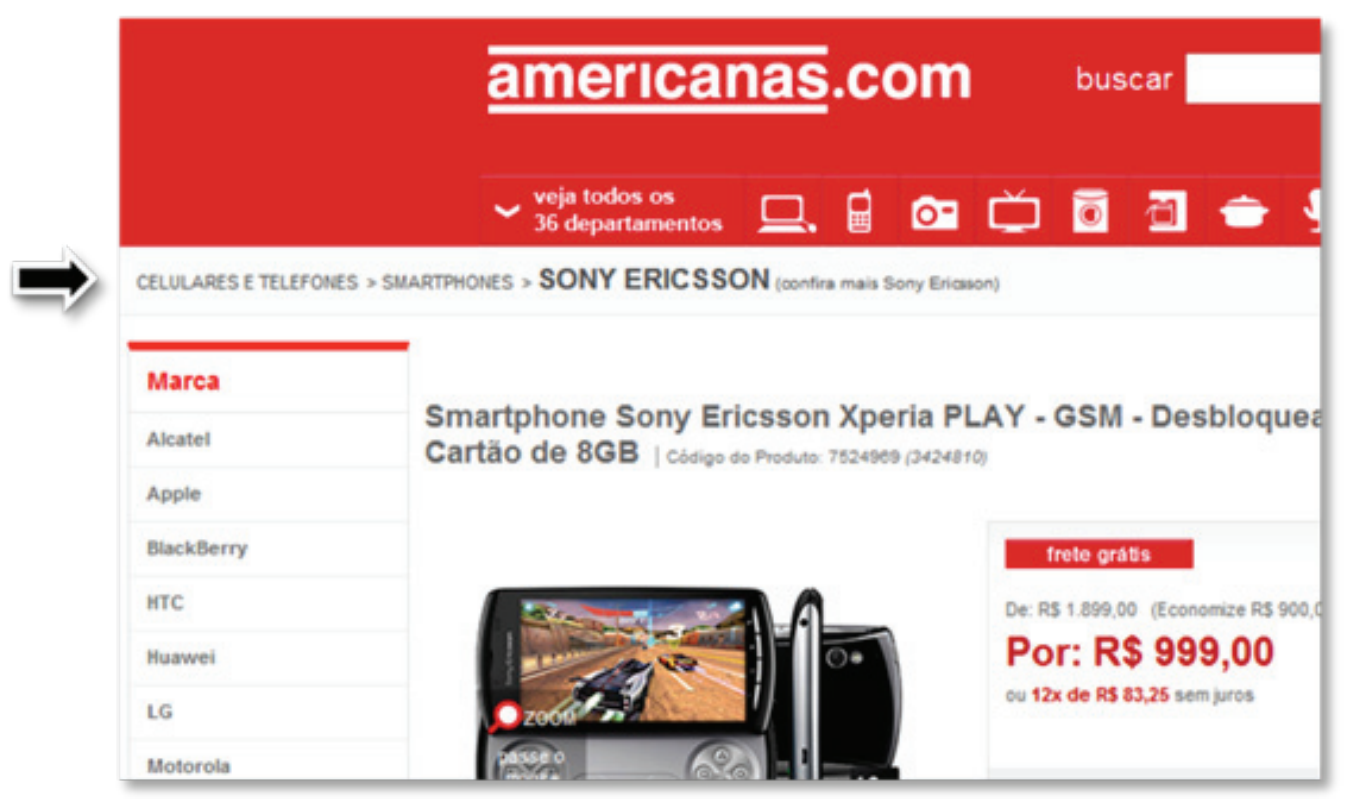

Figura 7 Opção breadcrumbs (Fonte: http://www.americanas.com.br (acesso em 17 de abril de 2012)

Sobre os recursos audiovisuais, verificou-se que imagens estáticas e/ou ícones, imagens dinâmicas, animações e/ou vídeo, texto estático e elementos esquemáticos foram encontrados na interface de todos os sites analisados. Apenas o texto dinâmico ( 5 de 11) e som (4 de 11) foram apresentados em poucos sites da amostra.

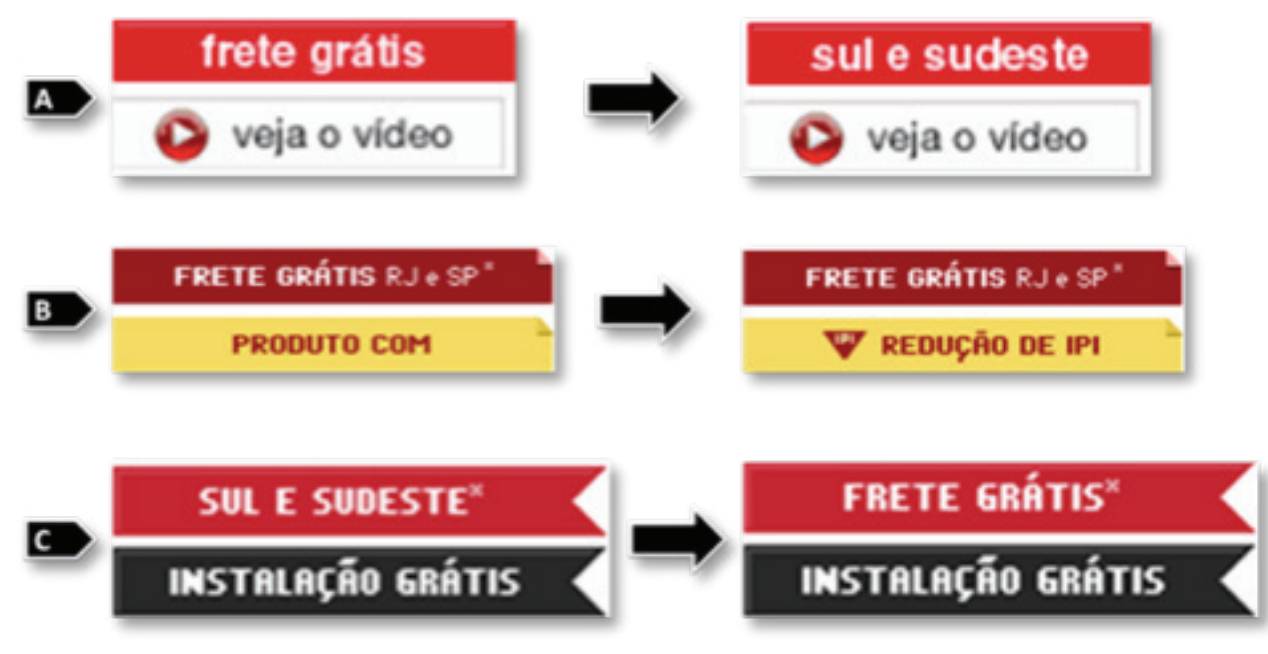

Figura 8 Exemplos de texto dinâmico

Exemplo A: Texto dinâmico da opção frete grátis - http://www. americanas.com.br (12/11/12)

Exemplo B: Texto dinâmico informando redução de IPI - http://www. pontofrio.com.br $(12 / 11 / 12)$

Exemplo C: Frete grátis para determinados estados - http://www.extra. com.br $(12 / 11 / 12)$ 
Após a análise gráfico-informacional, foi possível identificar outras tendências presentes nos sites analisados e com isso, acreditamos que os sites utilizam recursos gráfico-visuais em uma maior quantidade nos estágios iniciais do processo da compra. Os itens de recursos audiovisuais são mais evidenciados nos estágios da página inicial e na seção do produto.

Em contraste, o site Voegol não apresentou vários itens da análise, motivo pelo qual acreditamos que seus serviços não estão direcionados e estruturados da mesma forma que os outros sites de comércio eletrônico. Por exemplo, para a opção de carrinho de compras, no site Voegol o e-consumidor compra apenas uma passagem aérea, um produto em si e não vários como pode ser comprado nos outros sites.

Durante a análise registramos algumas peculiaridades, por exemplo, com o crescimento das redes sociais, os sites da amostra tiveram que adaptar-se e estão disponibilizando acesso e compartilhamento nas redes sociais do e-consumidor. Podemos constatar também, que quando se trata de um produto novo e seja investido um marketing de propaganda nele, os sites disponibilizam página especial com detalhamentos e recursos audiovisuais do lançamento do produto no mercado, para o e-consumidor acessar e ficar por dentro da novidade.

Notou-se também, uma poluição visual na etapa da página inicial, uma grande quantidade de informações textuais e gráficas que podem prejudicar as escolhas e o andamento do processo da compra online. Todavia, não sabemos ao certo até que ponto o e-consumidor se adaptou ou não a esta quantidade excessiva de informação.

Também, notamos que alguns sites da amostra prezam pela carga de trabalho, reduzindo ao máximo a quantidade de páginas no processo (destaque para a etapa da finalização da compra), mas, até que ponto a redução pode facilitar o entendimento e as ações que e-consumidor precisa realizar durante o processo da compra online, ainda carece de maior investigação.

\section{Conclusão}

Da análise dos resultados, podemos concluir que houve uma ênfase nas mudanças do processo da compra. Ao realizar a comparação com os estudos de O'Connel (2002), Miranda (2004) e Junior (2006), constatamos mudanças expressivas nos estágios que compõem a compra online atual. Os resultamos mostraram que os estágios iniciais ainda são apresentados na mesma forma, porém ao iniciar o checkout, o e-consumidor encontrará diversidade no processo da compra online em B2C. Assim, podemos concluir que os estágios que formam o processo da compra em sites $\mathrm{B} 2 \mathrm{C}$, sofreram mudanças na sua estrutura, alguns destes estágios foram mesclados, outros mudaram de posição, foram alterados em sua sequência e alguns já não existem mais no processo atual. Além disso, o processo apresentou ordem 
diferenciada dos estágios, estágio facultativo e ainda diferenciação por gênero específico de B2C.

No que diz respeito à análise gráfico-informacional, podemos dizer que recursos audiovisuais são mais evidenciados nos estágios iniciais. Após a página inicial e seção do produto os componentes se tornaram mais simplificados na aparência visual da interface B2C. Sobre as informações, os itens considerados necessários para navegar, foram apresentados na maioria dos sites analisados. Assim, podemos dizer, com base na literatura, que as informações apresentadas ao e-consumidor atendem as necessidades e preenchem os requisitos necessários para navegar em um site B2C.

Cumpre ressaltar que na finalização da compra houve uma redução dos itens informacionais recomendados pela literatura, principalmente na diferenciação que encontramos durante o checkout. A interface B2C sofreu recentemente mudanças visuais e informacionais durante a finalização da compra nos sites da amostra, por isso, alguns sites B2C apresentaram diferentes informações e estruturas nas interfaces que compreendem os estágios finais do processo da compra.

Também podemos concluir que houve defasagem nos itens da análise no site Voegol (compra de passagens aéreas), essas alterações comprovam que os serviços oferecidos ao e-consumidor assim como o conteúdo, funcionalidades e estrutura de navegação estão vinculados também ao gênero de B2C.

Dos outros sites da amostra, podemos dizer que as informações e elementos gráficos que compõem a interface $\mathrm{B} 2 \mathrm{C}$ atendem às necessidades primordiais para realizar a compra online.

De maneira geral, podemos concluir que o comércio eletrônico B2C sofre constantes modificações, a interface é mutável. Porém, até que ponto as alterações, mudanças feitas pelos desenvolvedores afetam o comportamento do e-consumidor durante a interação? Os desenvolvedores consideram ao implementar mudanças constantes das interfaces $\mathrm{B} 2 \mathrm{C}$ o fator comportamental do e-consumidor?

Como desdobramento deste estudo, realizaremos um ensaio de interação em sites B2C, com o intuito de verificar quais aspectos da interface motivam ou desmotivam os e-consumidores brasileiros a realizar compras online.

\section{Referências}

ALbertin, A. L. (2010). Comércio eletrônico: modelo, aspectos e contribuições de sua aplicação. 6⿳a ed. São Paulo: Editora Atlas S. A.

BRASPAG. (2012). Transações no e-commerce crescem 46\% em 2012. Disponivel em: <http://www.braspag.com.br/cat/noticias/>. Acesso em: 18 Dezembro 2012.

CYBIS, W. D. A. (2003). Engenharia de usabilidade: Uma abordagem ergonômica. Apostila do laboratório de utilizabilidade de informática - UFSC.

Florianópolis, p. 1-138. 
CYBIS, W.; BETIOL, A. H.; FAUST, R. (2007). Ergonomia e usabilidade: conhecimentos, métodos e aplicações. São Paulo: Novatec Editora Ltda.

E-BIT. (2012). WebShoppers $26^{a}$ edição. E-bit Empresa \& Câmara Brasileira de Comércio Eletrônico. São Paulo, p. 1-40.

FLEMING, J. (1998). Web Navigation - Designing the User Experience. $1^{\mathrm{a}}$ ed. Sebastopol, California: O’Reilly \& Associates Inc.

GALITZ, W. O. (2002). The essential guide to user interface design: An introduction to GUI design principles and techniques. $2^{\text {a }}$. ed. New York: John Wiley \& Sons.

HACKOS, J. T.; REDISH, J. C. (1998). User and task analysis for interface design. New York: John Wiley \& Sons, Inc.

INFO Online. Notícias/Mercado: E-commerce crescerá 35\% em 2013 no Brasil, diz IDV, 2012. Disponível em: <http://info.abril.com.br/noticias/mercado/ecommerce-crescera-35-em-2013-no-brasil-diz-idv-17122012-44.shl?utm_ source=feedburner\&utm_medium=feed\&utm_campaign=Feed\%3A+PlantaoINFO+\%28Plant\%C3\%A30+INFO\%29>. Acesso em: 18 Dezembro 2012. JUNIOR, S. D. D. L. (2006). Modelo conceitual de desenvolvimento de sistema informacional para e-commerces brasileiros. 2006. 295 f. Dissertação apresentada à Universidade Federal de Pernambuco, Centro de Artes e Comunicação, Programa de pós graduação em Design - Mestrado em Design, Área de concentração: Design e ergonomia, Recife, Fevereiro.

KAlBaCh, J. (2007). Designing Web Navigation. $1^{\text {a }}$ ed. Sebastopol/California: O'Reilly Media Inc.

LANSDALE, M. W.; ORMEROD, T. C. (1994). Understanding interfaces: A handbook of human-computer dialogue. London: Academic Press.

MATHWORKs. (2012). Creating Graphical User Interfaces (versão: 2012b). The MathWorks, Inc. Natick, Massachusetts, p. 1-179.

MIRANDA, F. M. P. D. (2004). Estudo ergonômico de websites de comércio eletrônico: seleção do produto pelo usuário no processo de compra. 2004. $314 \mathrm{f}$. Dissertação de mestrado - Programa de Pós-Graduação do Departamento de Artes e Design - Pontifícia Universidade Católica do Rio de Janeiro, Rio de Janeiro.

o'CONNEL, в. (2002). B2B.com - Ganhando dinheiro no e-commerce business-tobusiness. Tradução de Seagull Translation Services e Martha Malvezzi Leal. São Paulo: Makron Books Ltda.

osório, J. (2011). Tendências do E-Commerce em 2012 no Brasil. E-Commerce, 2011. Disponível em: <http://ecommercenews.com.br/artigos/cases/tendenciasdo-e-commerce-em-2012-no-brasil>. Acesso em: o8 dezembro 2011.

PADOVANI, S.; SPINILlo, C. G.; GOMES, Í. M. D. A. (2009). Desenvolvimento e aplicação de modelo descritivo-normativo para análise de websites. Produção, v. 19, n. 3, p. 514-528, Setembro/Dezembro 2009.

QIN, z. (2009). Introduction to E-commerce. New York: Tsinghua University Press, Beijing \& Springer Berlin Heidelberg.

Robbins, J. N. (2006). Web Design in a Nutshell: A Desktop Quick Reference. $3^{\text {a }}$. ed. Sebastopol: O’Reilly Media, Inc. ISBN 0-596-00987-9.

STECANElla, v. (2013). Economia\&Negocios - Estadão.com.br. Disponível em: $<$ http://economia.estadao.com.br/noticias/economia+geral,vendas-onlinedevem-crescer-25-em-2013-preve-e-bit,140864,o.htm>. Acesso em: 23 de janeiro de 2013. 
TASSABEHJI, R. (2003). Applying e-commerce in business. University of Bradford West Yorkshire: Sage Publications Ltd.

TIDWELL, J. (2011). Designing Interfaces. 2ª . ed. Sebastopol: O’Reilly Media, Inc.

ISBN 978-1-449-37970-4.

\section{Sobre os autores}

\section{Stephania Padovani,}

$<$ s_padovani2@yahoo.co.uk>

Professora e pesquisadora do Departamento de Design da UFPR. Tem experiência nas áreas de Interação Humano-Computador e Ergonomia, atuando nos seguintes temas: hipermídia, sistemas de navegação, avaliação de usabilidade e design de interfaces.

\section{André Schlemmer,}

$<$ schlemmer.andre@gmail.com>

Mestrando do Programa de Pós-Graduação em Design pela UFPR (na linha de pesquisa de Design de Sistemas de informação). Seus principais temas de interesse são: interação humano-computador, design centrado no usuário e experiência do usuário.

Artigo recebido em o9 dez. 2013, aprovado em 17 dez. 2013. 


\section{Apêndice: Modelo de análise gráfico-informacional em comércio eletrônico B2C}

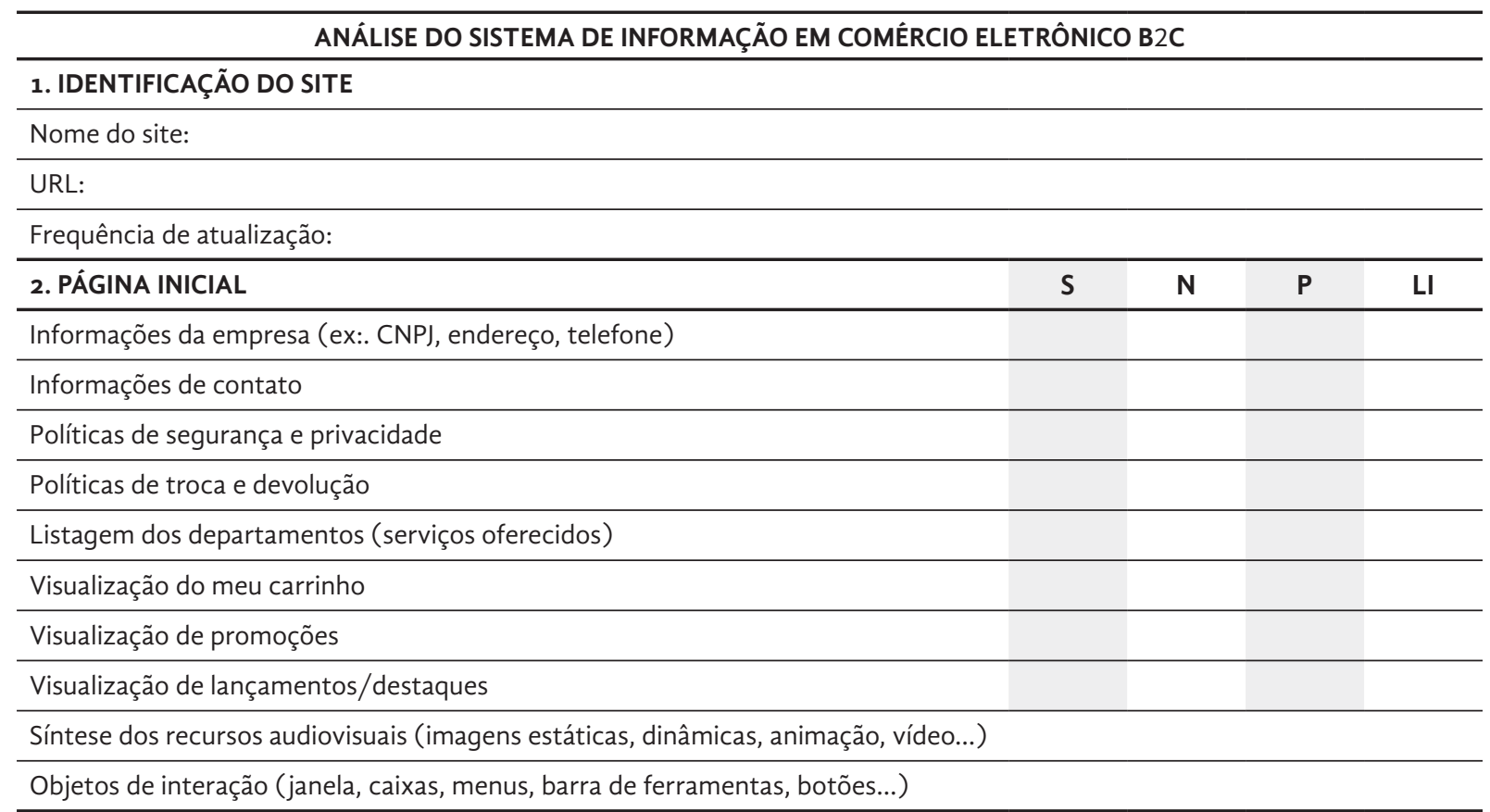

\begin{tabular}{|c|c|c|c|c|}
\hline 3. SEÇÃO DO PRODUTO & S & $\mathbf{N}$ & $\mathbf{P}$ & LI \\
\hline \multicolumn{5}{|c|}{ Visualização do produto em conjunto com detalhes e preço } \\
\hline \multicolumn{5}{|l|}{ Informações técnicas avançadas sobre o produto } \\
\hline \multicolumn{5}{|l|}{ Custo de frete durante a seleção do produto } \\
\hline Prazo de entrega durante a seleção do produto & & & & \\
\hline
\end{tabular}

Síntese dos recursos audiovisuais (imagens estáticas, dinâmicas, animação, vídeo, menu interativo...)

Objetos de interação (janela, caixas, menus, barra de ferramentas, botões...)

\begin{tabular}{|c|c|c|c|c|}
\hline 4. CARRINHO DE COMPRAS & S & $\mathbf{N}$ & $\mathbf{P}$ & LI \\
\hline \multicolumn{5}{|l|}{ Visualização dos dados do produto adicionado } \\
\hline \multicolumn{5}{|l|}{ Visualização do preço unitário e do valor total de cada produto } \\
\hline \multicolumn{5}{|l|}{ Listagem dos produtos adicionados } \\
\hline \multicolumn{5}{|l|}{ Identificação de descontos fornecidos } \\
\hline \multicolumn{5}{|l|}{ Custo final da compra } \\
\hline \multicolumn{5}{|c|}{ Síntese dos recursos audiovisuais (imagens estáticas, dinâmicas, animação, vídeo, menu interativo...) } \\
\hline \multicolumn{5}{|l|}{ Objetos de interação (janela, caixas, menus, barra de ferramentas, botões...) } \\
\hline 5. FINALIZAÇÃO DA COMPRA & S & $\mathbf{N}$ & $\mathbf{P}$ & LI \\
\hline \multicolumn{5}{|l|}{ Visualização da linha do tempo ao e-consumidor } \\
\hline \multicolumn{5}{|l|}{ Forma de pagamento } \\
\hline \multicolumn{5}{|l|}{ Tipos de entrega } \\
\hline \multicolumn{5}{|l|}{ Prazo de entrega } \\
\hline \multicolumn{5}{|l|}{ Garantia do produto } \\
\hline \multicolumn{5}{|l|}{ Resumo dos produtos comprados em conjunto com o custo final da compra } \\
\hline $\begin{array}{l}\text { Todas as informações (dados pessoais, endereço de entrega, valores e forma de } \\
\text { pgto) para confirmação da compra }\end{array}$ & & & & \\
\hline
\end{tabular}


Schlemmer, A. \& Padovani, S. | Estudo analítico dos estágios e informações que compõem a compra online

Síntese dos recursos audiovisuais (imagens estáticas, dinâmicas, animação, vídeo, menu interativo...)

Objetos de interação (janela, caixas, menus, barra de ferramentas, botões...)

$\mathbf{S}=\operatorname{Sim}|\mathbf{N}=\mathbf{N a ̃ o}| \mathbf{P}=$ Parcial $\mid \mathrm{LI}=$ Localização inadequada 